\title{
Out-of-hospital cardiac arrest survival in Athens: Data from a Greek public hospital
}

\author{
Zisimangelos S. Solomos, ${ }^{1}$ Maria S. Tatsi, ${ }^{2}$ Victoria E. Psomiadou, ${ }^{3}$ Angeliki D. Tsifi, ${ }^{4}$ \\ Dimitrios S. Theodoridis, ${ }^{5}$ Aikaterini P. Petropoulou, ${ }^{6}$ Konstantinos G. Miltiadou ${ }^{7}$
}

'Internal Medicine, 'Medecins du Monde', Greek Delegation, Athens; 'Department of General Surgery, Pammakaristos General Hospital, Athens; ${ }^{3}$ Department of Gynaecological Oncology, Metaxa Memorial Cancer Hospital, Piraeus; ${ }^{4}$ Intensive Care Unit, Konstantopouleio-Patision General Hospital, Athens; ${ }^{5}$ Department of Haematology, Konstantopouleio-Patision General Hospital, Athens; ' Dental School, University of Athens, Athens; ' Hepatogastroenterology Unit, 2nd Department of Internal Medicine Propaedeutic, Research Institute and Diabetes Center, Medical School, National and Kapodistrian University, Attikon University General Hospital, Athens, Greece

\begin{abstract}
Although out-of-hospital cardiac arrests (OHCA) are a major cause of mortality internationally, data regarding survival in Greece remains scarce and inconclusive. The aim of this study is to assess the immediate and 24-hour survival of OHCA sufferers during a 5-year period in a public hospital in Athens. A retrospective study was conducted on all cardiac arrests that were transferred to our hospital during a five-year period (2011-2015). Our primary objective was to calculate return of spontaneous circula-
\end{abstract}

Correspondence: Zisimangelos S. Solomos, Internal Medicine, 'Medecins du Monde', Greek Delegation, Dimitrakopoulou str. 130, P.C. 11741, Koukaki Area, Athens, Greece.

Tel: +302103809899 .

E-mail: zisimosmed@gmail.com

Key words: Out-of-hospital cardiac arrest; Cardiopulmonary resuscitation; Mortality; Greek hospital.

Contributions: ZSS and KGM authored and edited the manuscript; ADT, VEP, MST, APP, DST searched and extracted data from medical files. ZSS and APP analysed the data

Conflict of interest: the authors declare no potential conflict of interest.

Funding: none.

Conference presentation: data from the study were submitted as two poster presentations at the 2016 World Congress of Internal Medicine (22-25 August, Bali, Indonesia) and at the 2016 European Resuscitation Congress (24-25 September, Reykjavik, Iceland).

Received for publication: 24 April 2019.

Revision received: 3 June 2019

Accepted for publication: 6 June 2019.

This work is licensed under a Creative Commons Attribution 4.0 License (by-nc 4.0)

(C) Copyright: the Author(s), 2019

Licensee PAGEPress, Italy

Emergency Care Journal 2019; 15:8249

doi:10.4081/ecj.2019.8249 tion (ROSC) and 24-hour survival ratios. Our secondary objective was to estimate 30-day survival. Demographic data was also collected. 283 OHCA were included in the study. The mean age was 67.2 years and the male: female ratio was 2 . Medical history was available for $33.6 \%$ of patients. $72.1 \%$ of cases were transferred to the emergency department by ambulance and $2.8 \%$ by private means of transport. Cardiopulmonary resuscitation was attempted on $57.6 \%$ of cardiac arrests, $8.6 \%$ regained ROSC and $6.1 \%$ survived for 24 hours. The 30-day survival was expected to be less than 3.5\%. In our institution, ROSC, 24-hour and expected 30-day survival were lower than the European average and in accordance with the recent prospective Eureca One study. However, data from our institution cannot be generalised and multicenter studies are required in order to clarify OHCA outcomes in Greece.

\section{Introduction}

Out-of-hospital cardiac arrest (OHCA) is a major cause of mortality internationally, with 275,000 and 420,000 annual deaths in Europe and the United States respectively. ${ }^{1}$ Data regarding the survival of OHCA victims in Greece remain scarce and mostly derive from a handful of single-center studies ${ }^{2,3}$ that do not estimate 30-day survival or survival to hospital discharge, in contrast with similar studies from other European Union (EU) countries. ${ }^{4}$ Additionally, although the superiority of cardiopulmonary resuscitation (CPR) certified from uncertified medical personnel in increasing OHCA survival has been well described in regional studies, ${ }^{2,5}$ inadequacies in CPR skills of the medical and nursing staff in various Greek hospitals, especially in institutions with relatively small incidence of cardiac arrests, ${ }^{6}$ have been reported in the literature..$^{7-10}$

The aim of this study is to assess the immediate and 24-hour survival of OHCA victims, as well as CPR efficiency of healthcare personnel during a 5-year period in a public hospital emergency department (ER) in Athens, in order to increase the available data regarding OHCA mortality in Greece.

\section{Materials and Methods}

A retrospective study was conducted on all OHCA victims that were transferred to the ER of our institution (Pammakaristos General Hospital of Athens), during a five-year period (20112015). Our hospital is a middle-sized public general hospital, with approximately 170 beds that is located in a densely populated area 
of the (Kato Patisia) center of Athens. It is on call every 4 days and, along with 2 middle-sized (Konstantopouleio, Amalia Fleming) and 3 larger (Giorgos Gennimatas, Tzaneio, Sotiria) tertiary hospitals, ${ }^{11}$ covers the emergencies for the entire urban population of Athens (approximately 3 million). ${ }^{12}$

Upon occurrence of an OHCA, the dispatched ambulance contacts the hospital several minutes before arriving to the ER, in order for the resuscitation team to be assembled. The latter usually consists of the (resident or senior) internist, cardiologist and anesthesiologist on call, as well as at least one nurse. The CPR is provided in a specifically equipped resuscitation room in the ER and commences immediately upon arrival of the ambulance. If return of spontaneous circulation (ROSC) occurs, the patient is admitted to one of the hospital's advanced care units (total capacity of six beds) for monitoring. The national Emergency Medical Services (EMS/EKAV) center is then contacted and the survivor is registered as a tertiary hospital intensive care unit (ICU) candidate. In case of ICU bed availability, the EMS center arranges for the transfer of the patient with an ambulance. In case of bed unavailability, the registration of the survivor is renewed every 24 hours by the current hospital's doctor on call.

After the submission of the research protocol and the approval from the institution's scientific and administrative committees, access to the hospital's archive was granted. All OHCA entries in the ER patient registry for the years 2011-2015 were collected. The data acquired, included date of ER admission, sex, age, means of transport to the hospital (ambulance or private), whether CPR was provided to the victim, outcome of CPR (ROSC or death) and where available, medical history. In case of ROSC and hospital admission, the patients' medical records were collected from the hospital's archive to assess 24-hour survival.

Our primary objective was to calculate OHCA immediate (ROSC) and 24-hour survival ratios. Our secondary objectives were to calculate ROSC per CPR attempt ratio and to estimate 30day survival and survival to hospital discharge.

Cardiac arrest victims above 90 years of age or with a history of generalized neoplastic disease were excluded from the study, as benefit from CPR on those patients was already expected to be insignificant. ${ }^{13-17}$

\section{Results}

During the 5-year period of the study, 301 OHCA were transferred to the hospital's ER. 6\% (18) were excluded from the dedicated part of the study because they met the exclusion criteria (above 90 years of age, generalized neoplastic disease) thus reducing the study population to 283 cases.

The mean age of the patients was 67.2 years and the male: female ratio was 2 . Medical history was documented for only $33.6 \%$ (95) of OHCA victims, with $32.6 \%$ (31) of them having coronary artery disease (Table 1). $72.1 \%$ (204) of cases were transferred to the ER by ambulance, $2.8 \%$ (8) were transferred by private means of transport, while in $25.1 \%$ (71) of the cases the means of transport was not documented.

CPR was attempted on $57.6 \%$ (163) of cardiac arrests. No survival benefit was expected from CPR provision for the rest $42.4 \%$ (120) of the cases (prolonged cardiac arrest with no bystander or EMS personnel provision of CPR for $>15$ min, extensive delay of ambulance response and travel time to ER, presence of asystole for more than 30 min prior to ER transfer), thus CPR was not attempted by the hospital's medical personnel.
From those OHCA patients on whom CPR was performed, $8.6 \%$ (14) regained ROSC and 6.1\% (10) survived for 24 hours (Table 2 and Figure 1). The immediate ROSC and 24-hour survival rates for the whole 5-year period (including the total number of OHCA, irrespective of CPR provision or not) in our hospital were $5 \%$ and $3.5 \%$ respectively. The 30 -day survival and survival to hospital discharge ratios could not be calculated in this particular study, but can be expected to be equal to or less than the calculated 24-hour survival ratio $(3.5 \%)$.

\section{Discussion}

According to the data acquired from our institution during the 5 -year study period, initiation of resuscitation efforts by our arrest

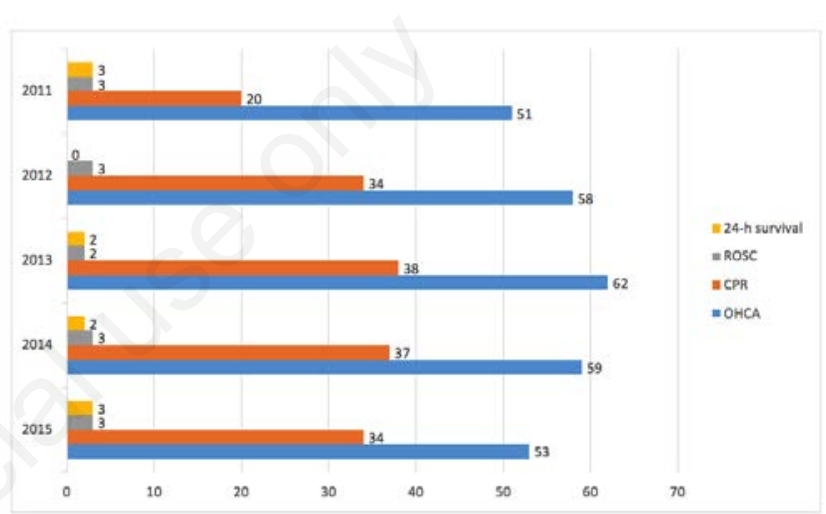

Figure 1. Out-of-hospital cardiac arrest (OHCA) victims, cardiopulmonary resuscitation (CPR) attempts, immediate and 24h survivors per year of study. ROSC, return of spontaneous circulation.

Table 1. Medical history of out-of-hospital cardiac arrests patients.

\begin{tabular}{lc} 
Patients with available history $(\mathrm{n=95})$ & 7 \\
Arterial hypertension & 4 \\
Chronic obstructive pulmonary disease & 2 \\
Central motor neuron disease & 2 \\
Congenital cardiac disease & 1 \\
\hline Aortic aneurysm & 4 \\
Alcohol or substance abuse & 7 \\
Presence of pacemaker & 31 \\
Coronary artery disease & 3 \\
Cardiomyopathies & 3 \\
Cardiac valvular disease & 10 \\
\hline Diabetes Mellitus & 2 \\
Stroke & 4 \\
No history & 15 \\
Other
\end{tabular}

Patients with undocumented history $(\mathrm{n}=188)$.

Total $(\mathrm{n}=283)$ 
Table 2. Summary data per year of study.

\begin{tabular}{lcccc} 
Year & OHCA $(\mathrm{n}=283)$ & CPR attempts $(\mathrm{n}=163)$ & 24-hour survivors (n=10) \\
2011 & 51 & 20 & 3 & 3 \\
2012 & 58 & 34 & 3 & 0 \\
\hline 2013 & 62 & 38 & 2 & 2 \\
2014 & 59 & 37 & 3 & 2 \\
\hline 2015 & 53 & 34 & 3 & 3 \\
\hline
\end{tabular}

OHCA, out-of-hospital cardiac arrests; CPR, cardiopulmonary resuscitation; ROSC, return of spontaneous circulation.

healthcare personnel was considered futile for $42.4 \%$ of OHCA victims that were transferred to the ER department, thus CPR was not attempted on them. Our findings are in good agreement with the Eureca One prospective study that included one-month OHCA outcome data from 27 European countries and was published in 2016. According to the study, in Greece, approximately $54 \%$ of patients either die on scene or are pronounced dead on arrival at hospital. ${ }^{1}$ A possible explanation for this excess mortality could be the long ambulance response and travel time to the ER, possibly as a result of the reduction of the Greek EMS ambulance fleet and its deficient maintenance due to the recent cuts in public health expenditure as a result of the ongoing financial crisis that adds up to an already inefficient EMS response mechanism. ${ }^{18}$ The unavailability of Automatic External Defibrillators in public areas ${ }^{19}$ and lack of civilian training on how to use them, as well as the low rates of bystander CPR could also be contributing factors. The latter is also implied in the Eureca One study which places Greece $25^{\text {th }}$ out of 27 countries in CPR provision (32 attempts per 100,000 arrests per year) by both bystanders and EMS personnel. ${ }^{1}$

The percentage of ROSC in patients on whom CPR was attempted (8.6\%) indirectly reflects this hospital ER personnel's CPR performance and is again in accordance with Eureca One's reported similar rate for Greece (9\%) which was the lowest among 27 countries. $^{1}$

Our study however carries several limitations:

- The 30-day mortality and the survival-to hospital discharge ratio, that are more accurate survival indexes, as well as the neurological outcome of the survivors could not be measured in this particular study. This is due to the hospital's policy of transferring long-term $(>24 \mathrm{~h})$ cardiac arrest survivors to tertiary hospital ICUs, making follow-up of the patients difficult. However, in our institution, the 30-day and overall survival for the 5 -year period of the study can be expected to be equal to or less than the calculated 24 -hour survival (3.5\% overall, $6,1 \%$ with CPR attempted) which is lower than the EU average ${ }^{4}$ and confirms Eureca One's 30-day survival estimates for Greece (less than $5 \%)^{1}$

- This is a retrospective, single-center study with a relatively small number of patients, thus inferences about nationwide OHCA survival in Greece cannot be drawn with certainty from the current data. In addition, this particular hospital's OHCA survival rate could be affected by certain institutional features (public general hospital and not specialized tertiary center, possible inadequate medical personnel's cardiac arrest training and experience, suboptimal composition of resuscitation team and local cardiac arrest protocols). Nevertheless, according to the country's National Healthcare System infrastructural features, the reference hospital is rather representative of the mean emergency care provider institution in Greece. 106 out of 124 public hospitals in Greece that admit emergencies are gen- eral hospitals, the majority of which are middle-sized (100-200 beds) that offer mostly secondary healthcare like our institution, while only 30 out of 124 public hospitals are either specialized centers (18) or large hospitals (more than 400 beds) that offer advanced tertiary care. ${ }^{20}$ The level of the CPR training of our institution's healthcare personnel could not be assessed in this particular study. However, given the fact that advanced life support (ALS) certification is not a prerequisite for a doctor to work in a Greek hospital ${ }^{20}$ and due to the inexistence of Emergency Care specialty in the Greek medical curriculum, ${ }^{21}$ we can presume that there are no major differences in CPR efficiency between the study's reference and other Greek hospitals, although circumstantial dissimilarities cannot be excluded.

- The extraction of the study's data in a period that represents the peak of Greece's economic recession and which featured major health expenditure cutbacks ${ }^{18}$ may not be indicative of pre or after crisis OHCA survival in our institution and could also be a limitation of the study.

\section{Conclusions}

In our institution, the immediate, 24-hour and expected 30-day survival were lower than the European average and in accordance with the recent prospective Eureca One study. Although, the results of this retrospective study cannot be generalized as they originate from a single centre, they contribute to the limited existing data regarding OHCA survival in our country. The conduction of similar efforts by other medical institutions as well as multicenter studies cannot be overemphasized in order to acquire a clearer picture regarding cardiac arrest outcomes in Greece. This could play an important role in the redesign of a more efficient EMS response mechanism.

\section{References}

1. Gräsner J-T, Lefering R, Koster RW, et al. EuReCa ONE, 27 Nations, ONE Europe, ONE Registry: A prospective one month analysis of out-of-hospital cardiac arrest outcomes in 27 countries in Europe. Resuscitation 2016;105:188-95.

2. Chalkias A, Koutsovasilis A, Mystrioti D, et al. Outcomes of cardiopulmonary resuscitation efforts in a Greek tertiary hospital. Acute Card Care 2013;15:34-7.

3. Galatianou I, Karlis G, Apostolopoulos A, et al. Body mass index and outcome of out-of-hospital cardiac arrest patients not treated by targeted temperature management. Am J Emerg Med 2017;35:1247-51.

4. Vargas M, Sutherasan Y, Pelosi P. Out-of-hospital cardiac 
arrest and survival to hospital discharge: a series of systemic reviews and meta-analyses. In: Vincent JL, ed. Annual update in intensive care and emergency medicine 2015. Switzerland: Springer International Publishing; 2015. pp 289-314.

5. Greif R, Lockey AS, Conaghan P, et al. European Resuscitation Council Guidelines for Resuscitation 2015: Section 10. Education and implementation of resuscitation. Resuscitation 2015;95:288-301.

6. Passali C, Pantazopoulos I, Dontas I, et al. Evaluation of nurses' and doctors' knowledge of basic \& advanced life support resuscitation guidelines. Nurse Educ Pract 2011;11:365-9.

7. Pantazopoulos I, Aggelina A, Barouxis D, et al. Cardiologists' knowledge of the 2005 American Heart Association Resuscitation Guidelines: The Athens Study. Heart Lung J Crit Care 2011;40:278-84.

8. Kyriakou F, Iacovidou N, Garofalakis I, et al. Residents' resuscitation training and theoretical knowledge in a Greek General Hospital. Eur J Emerg Med 2011;18:34-7.

9. Plagisou L, Tsironi M, Zyga S, et al. Assessment of nursing staff's theoretical knowledge of cardiovascular resuscitation in an NHS public hospital. Hell J Cardiol 2015;56:149-53.

10. Xanthos T, Akrivopoulou A, Pantazopoulos I, et al. Evaluation of nurses' theoretical knowledge in Basic Life Support: a study in a district Greek hospital. Int Emerg Nurs 2012;20:28-32.

11. Ministry of Health. Hospitals on call. 2019. Available from: http://www.moh.gov.gr Accessed: 2 June 2019.

12. Hellenic Statistical Authority. 2011 Census. 2011. Available from: http://www.statistics.gr/en/home/ Accessed: 2 June 2019.

13. Swor RA, Jackson RE, Tintinalli JE, Pirrallo RG. Does advanced age matter in outcomes after out-of-hospital cardiac arrest in community-dwelling adults? Acad Emerg Med 2000;7:762-8

14. Funada A, Goto Y, Maeda T, et al. Improved survival with favorable neurological outcome in elderly individuals with out-of-hospital cardiac arrest in Japan - A nationwide observational cohort study. Circ J 2016;80:1153-62.

15. Fukuda T, Ohashi-Fukuda N, Matsubara T, et al. Trends in outcomes for out-of-hospital cardiac arrest by age in Japan: an observational study. Medicine (Baltimore) 2015;94:e2049.

16. Libungan B, Lindqvist J, Strömsöe A, et al. Out-of-hospital cardiac arrest in the elderly: A large-scale population-based study. Resuscitation 2015;94:28-32.

17. Bossaert LL, Perkins GD, Askitopoulou H, et al. European Resuscitation Council Guidelines for Resuscitation 2015: Section 11. The ethics of resuscitation and end-of-life decisions. Resuscitation 2015;95:302-11.

18. Kotsiou OS, Srivastava DS, Kotsios P, et al. The emergency medical system in Greece: opening Aeolus' bag of winds. Int J Environ Res Public Health 2018;15-745.

19. ToVima team. Application helps you find location of available public AEDs. Greece: ToVima; 2017. Available from: https://www.tovima.gr/2017/10/18/society/app-sas-boitha-nabreite-poy-yparxoyn-aytomatoi-ekswterikoi-apinidwtes/ Accessed: 3 April 2019.

20. Economou C, Kaitelidou D, Karanikolos M, Maresso A. Greece: Health system review. Health Systems in Transition 2017;19:1-192.

21. Athens Doctor's Association. Establishing and renaming of medical specialties. 2018. Available from: http://www.isathens.gr/images/eggrafa/FEK-metonomasia-eidikotitwn4138.pdf Accessed: 2 June 2019. 